The Egyptian Journal of Hospital Medicine (January 2019) Vol. 74 (3), Page 575-583

\title{
Visual Acuity, Rafraction, And K Reading Changes After Five Versus Six Millimeters Optical Diameter Intracorneal Ring Segments For Treatment Of Keratoconus
}

\author{
*Adel M. Abdul Wahab, *Mohammed I. El-kasaby, *Mohammed T. Elnaggar, *Shaimaa M. \\ Mostafa \\ *Faculty of medicine for girls, Al Azhar University** Cario- Egypt- Research institute of \\ ophthalmology. \\ Corresponding author:Shaimaa Mamdouh Mostafa,email: sh_mesallam3@yahoo.com,Mobil :+01094142798
}

\begin{abstract}
Purpose: To evaluate changes in visual acuity, refraction, and keratometric $(\mathrm{K})$ readings after implantation of five millimeters versus six millimeters optical diameter of intracorneal ring segments (ICR) for treatment of keratoconus grade (2-4).

Patients and methods: A prospective nonrandomized clinical comparative study. Thirty four eyes were included in this study. They were classified into two groups:

Group I: Seventeen eyes of patients with Keratoconus grades (2-4) were subjected to $5 \mathrm{~mm}$ optical diameter intracorneal ring implantation.

Group II: Seventeen eyes of patient with keratoconus grade (2-4)were subjected to $6 \mathrm{~mm}$ optical diameter intracorneal ring implantation.

Results: Thirty four eyes of 22 patients (9 males and 13 females) with keratoconus grade (2-4) were enrolled in this study. The mean age \pm SD of the patients in group I was $26.36 \pm 7.06$ years and in group II was $23.09 \pm 6.92$ years.

The mean keratometric $(\mathrm{K})$ reading decreased from $50.05 \pm 3.64 \mathrm{D}$. to $46.96 \pm 4.42 \mathrm{D}$ in group I and from50.48 $\pm 5.18 \mathrm{D}$ to $46.91 \pm 5.44 \mathrm{D}$ in group II. There was a statistically significant improvement in the postoperative uncorrected distance visual acuity, corrected distance visual acuity, $\mathrm{K}$ readings, manifest spherical and cylindrical refractive errors, and spherical equivalent in both groups. No serious intraoperative complications were reported.

Conclusion: Both $5 \mathrm{~mm}$ and $6 \mathrm{~mm}$ ICR improve significantly UCVA, BCVA, decreased the corneal power and corneal astigmatism but $6 \mathrm{~mm}$ showed significant improvement in cylindrical errors and BCVA compared to $5 \mathrm{~mm}$ ICR.

Keywords: Corneal topography, Pentacam, femtosecond laser, keraring segment, keratoconus.

\section{Introduction}

Keratoconus $(\mathrm{Kc})$ is a progressive, noninflammatory, bilateral, ectatic disease of the cornea that is characterized by corneal steepening which progresses to irregular conical shape, with subsequent irregular astigmatism and decreased visual acuity ${ }^{(1)}$.

Intrastromal corneal ring segments (ICRS) were introduced as an alternative option for the refractive rehabilitation of patients with keratoconus, especially those with poor visual acuity not correctable with glasses and those with contact lens intolerance. One or two circular rings of (polymethyl methacrylate) were inserted in the mid peripheral corneal stroma. The net result was

anterior corneal surface, leading to improved refraction and visual acuity ${ }^{(2)}$.

Tunnel creation can be done either by a manual technique or using a femtosecond laser under topical anesthesia. The advent of the femtosecond laser has made the procedure safer, more accurate, and easier. Other advantages of femtosecond laser include less discomfort to the patient and better patient cooperation, precise control of tunnel depth, width, and centration ${ }^{(3)}$.

The aim of this study was to evaluate visual acuity, refraction, and topographic corneal changes after implantation of five millimeters versus six millimeters optical diameter of
\end{abstract} a flattening effect and regularization of the 
intracorneal ring segments for treatment of keratoconus.

\section{Patients and methods}

Thirty four eyes of 22 patients with keratoconus were enrolled in this nonrandomized prospective study.

This study was conducted in International eye hospital, and Ivision between April, 2015 and June, 2018.

The study protocol was adhered to the tents of the declaration of Helsinki and was approved by the ethical board of Al Azhar university. An informed consent was taken from each participant in the study.

Patients with a history of previous ocular surgery and coexisting ocular diseases other than keratoconus were excluded. Patients who failed to complete follow up examinations after surgery were also excluded.

The demographic data, material, and position of intracorneal ring, and ocular examination results, including measurements of uncorrected distance visual acuity (UDVA) and best-corrected distance visual acuity (BDVA), using automated chart projector (ACP.8; Topcon Corporation, Tokyo, Japan) were reported.

The refractive status was assessed using an auto refractometer (KR-800; Topcon Corporation, Tokyo, Japan). Intraocular pressure was measured by Goldman applanation tonometry (CT- 80; Topcon Corporation, Tokyo, Japan). Slit lamp examination and fundus evaluation were done by using an indirect ophthalmoscope.

Corneal indices were evaluated using the Pentacam (Oculus Pentacam; Optikgerate $\mathrm{GmbH}$, Wetzlar, Germany).

Inclusion criteria included age between 18 and 40 years, Maximum K reading less than $60 \mathrm{D}$ (based on Pentacam examination), and a central corneal thickness (CCT) of at least $400 \mu \mathrm{m}$.

Patients who had corneal scarring, any concomitant ocular disease, or any history of ocular surgery were excluded from the study. Patients who failed to complete follow-up examinations after the surgery were also excluded.

Patients were classified into two groups:

Group I: Seventeen eyes of patients with Keratoconus grade (2-4) were subjected to $5 \mathrm{~mm}$ optical diameter intracorneal ring implantation.

Group II: Seventeen eyes of patient with keratoconus grade (2-4) were subjected to $6 \mathrm{~mm}$ optical diameter intracorneal ring implantation. (KERARING Mediphacos Inc., Belo Horizonte, Brazil).

\section{Surgical technique}

Topical antibiotics were prescribed 2 days before surgery. The surgical decision about implant the intrastromal corneal rings was made according to the nomogram provided by the manufacturer (KERARING Mediphacos Inc., Belo Horizonte, Brazil).

After topical anesthesia $(0.5 \%$ propacaine hydrochloride eye drops), the geometric center of the cornea was marked.

A corneal tunnel was created with a femtosecond laser (Wavelight fs 200, Alcon Laboratories, Inc., Fort Worth, Taxes, USA).

A corneal tunnel was created at a depth of $75 \%$ of the corneal thickness at the thinnest location. (Wave length $1054 \mathrm{~nm}$, laser energy 1.5 mircojules, spot separation $6.5 \mu \mathrm{m}$ and frequency $200 \mathrm{kHz}$ ), The spot size of the laser beam was 5 $\mu \mathrm{m}$ in diameter. A tunnel for keraring implantation was created with the aid of the femtosecond laser. On optical zone (OZ) $5 \mathrm{~mm}$ in group (1) and on OZ 6mm in group (2) where the ICRS were to be inserted .After clearance of gas bubbles, a spatula was passed gently, and the intracorneal keraring segment was then implanted under full aseptic conditions using a special forceps, and was placed in the final position using a Sinskey hook. Topical antibiotics and a contact lens were applied.

After the procedure in both groups, topical antibiotics (for 1 week), steroids and lubricant eye drops (for 2 months) were prescribed.

Follow-up visits were scheduled at 1 day, 1,3 , and 6 , months after surgery. During followup visits, patients were subjected to UDVA and BDVA assessment, slit lamp examination, Pentacam and fundus examination.

\section{Statistical analysis:}

Statistical analysis was performed using the SPSS software version 19.0 (Statistical Package for Social Science). Data were presented as number, percentage, mean, standard deviation. Chi-square test and Fisher Excel test were used to compare between qualitative variables between two groups. Wilcoxon signed Rank test was done to compare quantitative variables between baseline and each time in case of non-parametric data. 
The level P-value considers statistically significant $<0.05$, High sig. $<0.001$ and not significant $>0.05$.

\section{Results}

Thirty four eyes of 22 patients (9 males; $40.91 \%$ and 13 females; 50.09\%) with keratoconus were enrolled in this study. Group 1 included 17 eyes of 11 patients (5 males $(54.5 \%)$ and 6 females $(45.5 \%)$ Their mean age \pm SD was $26.36 \pm 7.06$ years (Range $15-39$ years). Group 2 included 17 eyes of 11 patients (4 males, 36.4\% and 7 females, $63.6 \%$. Their mean age \pm SD was $23.09 \pm 6.92$ (Range 13-34 years). Statistically, the differences between both groups regarding age and sex were insignificant.

All patients completed the regular follow-up visits up to 6 months.

In Group I: The mean preoperative UCVA \pm SD was $0.05 \pm 0.04$. The mean preoperative $\mathrm{BCVA} \pm$ $\mathrm{SD}$ was $0.20 \pm 0.12$. It was improved at the end of follow-up visits postoperatively to $0.24 \pm 0.15$ (P 0.000), and $0.49 \pm 0.15$ (P 0.001) respectively. In Group II: The mean preoperative UCVA $\pm \mathrm{SD}$ was $0.06 \pm 0.05$ and the mean preoperative $\mathrm{BCVA} \pm \mathrm{SD}$ was $0.25 \pm 0.11$. It was improved at the end of follow-up visits postoperatively to 0.33 \pm 0.17 (p 0.000) and $0.70 \pm 0.23$ (P 0.000) respectively (Tables 1,2), (figures 1,4).

In Group I: The mean preoperative spherical error \pm SD was $-6.96 \pm 2.98 \mathrm{D}$. The mean preoperative cylindrical error \pm SD was $-4.69 \pm$ 1.58D. It was decreased at the end of follow-up visits postoperatively to $-3.21 \pm 2.28 \mathrm{D}$ ( $\mathrm{P} 0.000$ ) and $-2.51 \pm 0.97 \mathrm{D}$ (p 0.001) respectively. In Group II: The mean preoperative spherical error \pm SD was $-5.78 \pm 2.93 \mathrm{D}$ The mean preoperative cylindrical error $\pm \mathrm{SD}$ was $-5.07 \pm 1.61 \mathrm{D}$. It was decreased at the end of follow-up visits postoperatively to $-1.19 \pm 0.77 \mathrm{D}$. (P 0.000$)$ and $1.19 \pm 0.77 \mathrm{D}(\mathrm{P} 0.000)$ respectively (Figure 2,3). Group (I): The mean preoperative $\mathrm{K} 1 \pm \mathrm{SD}$ was $47.68 \pm 3.83 \mathrm{D}$. The mean preoperative $\mathrm{K} 2 \pm \mathrm{SD}$ was $52.77 \pm 3.82 \mathrm{D}$. The mean preoperative $\mathrm{Km} \pm$ $\mathrm{SD}$ was $50.05 \pm 3.64 \mathrm{D}$. It was decreased at the end of follow-up visits postoperatively to $45.56 \pm$ 4.30D (P 0.002), 48.54 $\pm 4.90 \mathrm{D}$. (P 0.001) and $46.96 \pm 4.42 \mathrm{D}$ (P 0.031) respectively. In Group (II): The mean preoperative $\mathrm{k} 1 \pm \mathrm{SD}$ was 48.50 \pm 4.67D . The mean preoperative $\mathrm{k} 2 \pm \mathrm{SD}$ was $52.51 \pm 5.65 \mathrm{D}$. The mean preoperative $\mathrm{km} \pm \mathrm{SD}$ was $50.48 \pm 5.18 \mathrm{D}$. which was decreased at the end of follow-up visits postoperatively to $45.86 \pm$ 5.49D (P 0.001), 48.01 \pm 5.44D (P 0.000) and $46.91 \pm 5.44 \mathrm{D}(\mathrm{P}$ 0.001) respectively (Figures $5,6,7)$.

One case in group $\boldsymbol{I}$ showed extrusion after blunt trauma 1 month after ring implantation. No complications were reported in group $\boldsymbol{I I}$.

Table 1. Parameters of group (I)

\begin{tabular}{|l|c|c|c|}
\hline Items & Preoperative & Postoperative & $\boldsymbol{p}$ \\
\hline UCVA & $0.05 \pm 0.04$. & $0.24 \pm 0.15$ & $(\mathrm{P} \mathrm{0.001)}$ \\
\hline Sphere & $-6.96 \pm 2.98 \mathrm{D}$. & $-3.21 \pm 2.28 \mathrm{D}$ & $(\mathrm{P} \mathrm{0.001)}$ \\
\hline Cylinder & $-4.69 \pm 1.58 \mathrm{D}$. & $-2.51 \pm 0.97 \mathrm{D}$. & $(\mathrm{p} 0.001)$ \\
\hline BCVA & $0.20 \pm 0.12$ & $0.49 \pm 0.15$ & $(\mathrm{P} \mathrm{0.001)}$ \\
\hline K 1 & $47.68 \pm 3.83 \mathrm{D}$. & $45.56 \pm 4.30 \mathrm{D}$. & (P 0.002) \\
\hline K2 & $52.77 \pm 3.82 \mathrm{D}$ & $48.54 \pm 4.90 \mathrm{D}$. & (P 0.001) \\
\hline Km & $50.05 \pm 3.64 \mathrm{D}$ & $46.96 \pm 4.42 \mathrm{D}$ & (P 0.031) \\
\hline
\end{tabular}

$\mathrm{NS}=$ nonsignificant level is considered at $\mathrm{P}$ value more than 0.05 . $\mathrm{S}=$ significant level is considered at $\mathrm{P}$ value less than 0.05 and $0.01 \mathrm{HS}=$ highly significant level is considered at $\mathrm{P}$ value less than 0.001 .

Table 2. Parameters of group (II)

\begin{tabular}{|l|c|c|c|}
\hline \multicolumn{1}{|c|}{ Items } & Preoperative & Postoperative & $\boldsymbol{p}$ \\
\hline UCVA & $0.06 \pm 0.05$. & $0.33 \pm 0.17$ & $(\mathrm{P} \mathrm{0.001)}$ \\
\hline Sphere & $-5.78 \pm 2.93 \mathrm{D}$. & $-2.74 \pm 2.89 \mathrm{D}$ & $(\mathrm{P} 0.001)$ \\
\hline Cylinder & $-5.07 \pm 1.61 \mathrm{D}$. & $-1.19 \pm 0.77 \mathrm{D}$. & $(\mathrm{p} 0.001)$ \\
\hline BCVA & $0.25 \pm 0.11$ & $0.70 \pm 0.23$ & $(\mathrm{P} \mathrm{0.001)}$ \\
\hline K 1 & $48.50 \pm 4.67 \mathrm{D}$. & $45.86 \pm 5.49 \mathrm{D}$. & $(\mathrm{P} 0.0001)$ \\
\hline K2 & $52.51 \pm 5.65 \mathrm{D}$ & $48.01 \pm 5.44 \mathrm{D}$. & $(\mathrm{P} 0.001)$ \\
\hline Km & $50.48 \pm 5.18 \mathrm{D}$ & $46.91 \pm 5.44 \mathrm{D}$ & (P 0.001) \\
\hline
\end{tabular}


Comparison between UVCA, sphere, $\mathrm{K} 1, \mathrm{~K} 2, \mathrm{Km}$ revealed statistically insignificant differences between both groups. However, cylindrical errors and BCVA revealed statistically significant differences between both groups, being more in group II.

$\mathrm{NS}=$ nonsignificant level is considered at $\mathrm{P}$ value more than 0.05 . $\mathrm{S}=$ significant level is considered at $\mathrm{P}$ value less than 0.05 and 0.01 .

$\mathrm{HS}=$ highly significant level is considered at $\mathrm{P}$ value less than 0.001 .

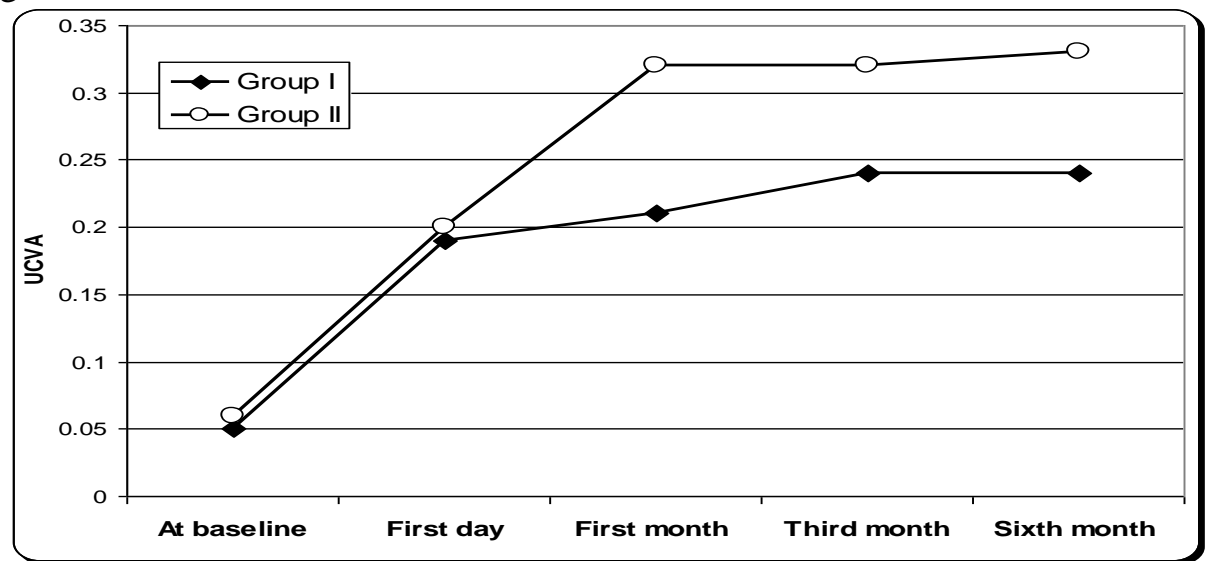

Figure (1). Correlation between UCVA after intracorneal ring implantation in both groups.

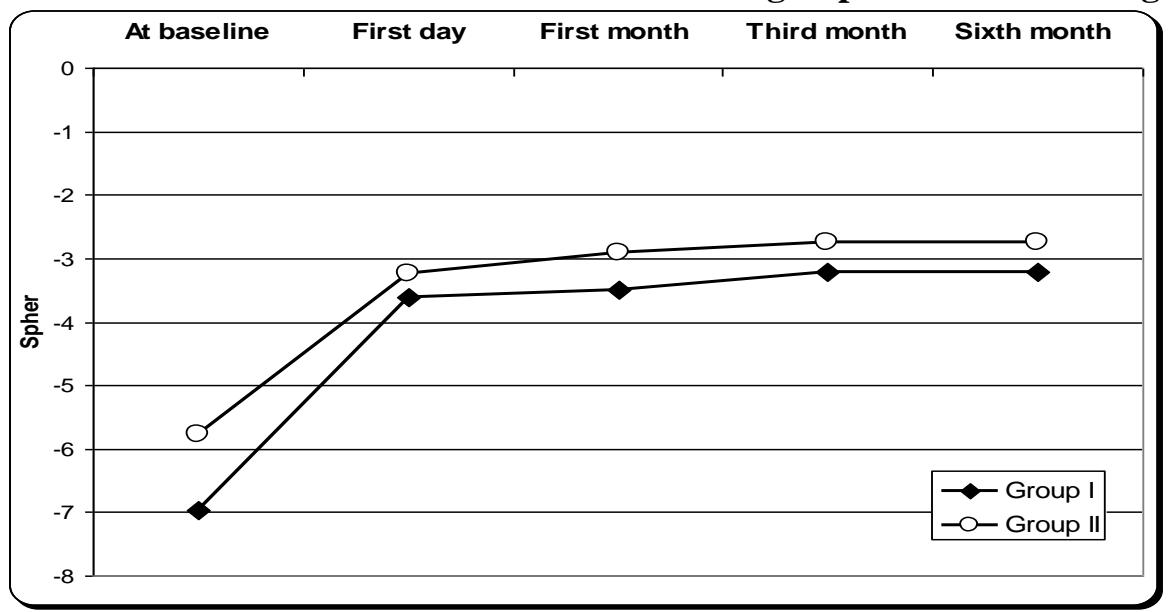

Figure (2). Correlation between spherical after intracorneal ring implantation in both groups.

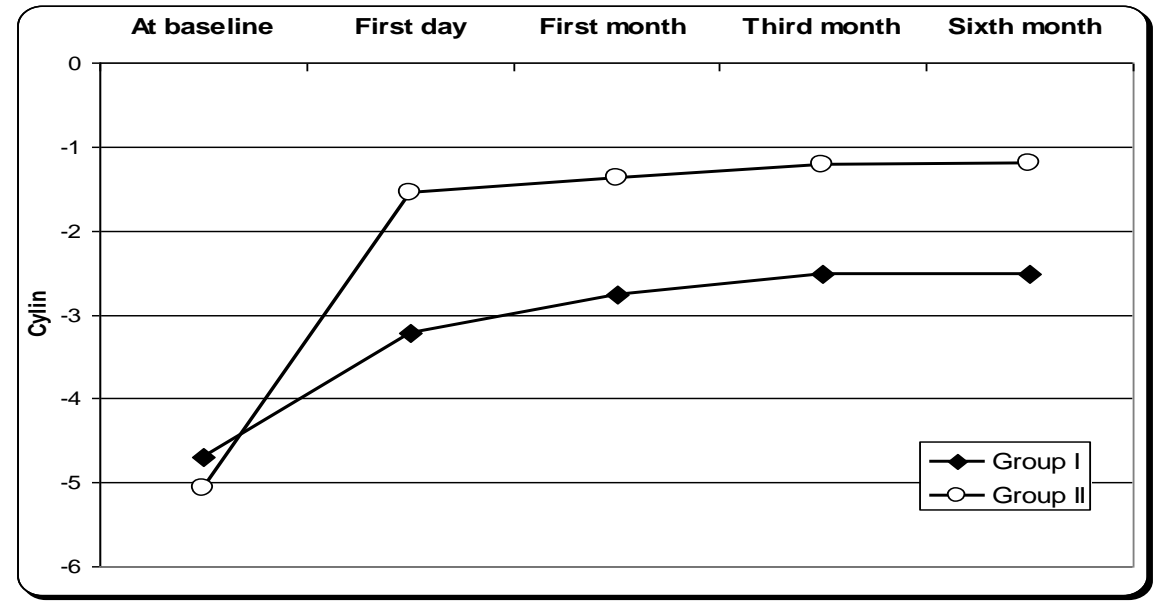

Figure (3). Correlation between cylindrical errors after intracorneal ring implantation in both groups. 


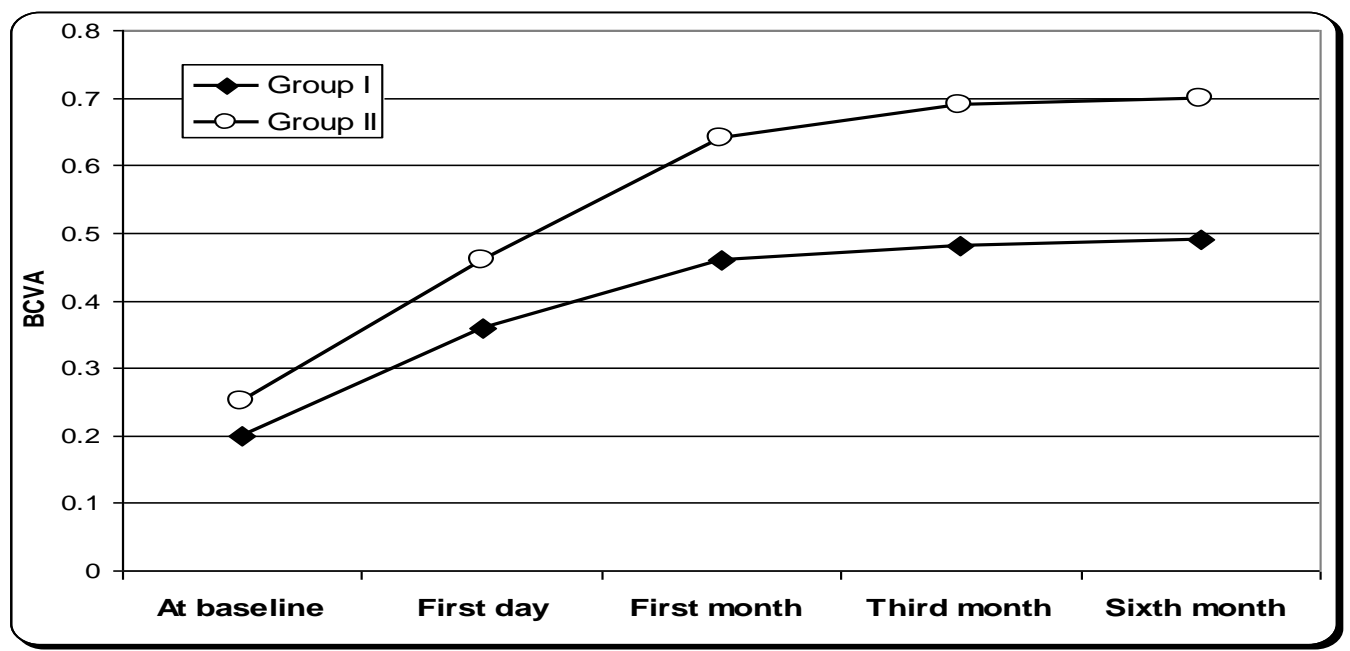

Figure (4). Correlation between BCVA after intracorneal ring implantation in both groups.

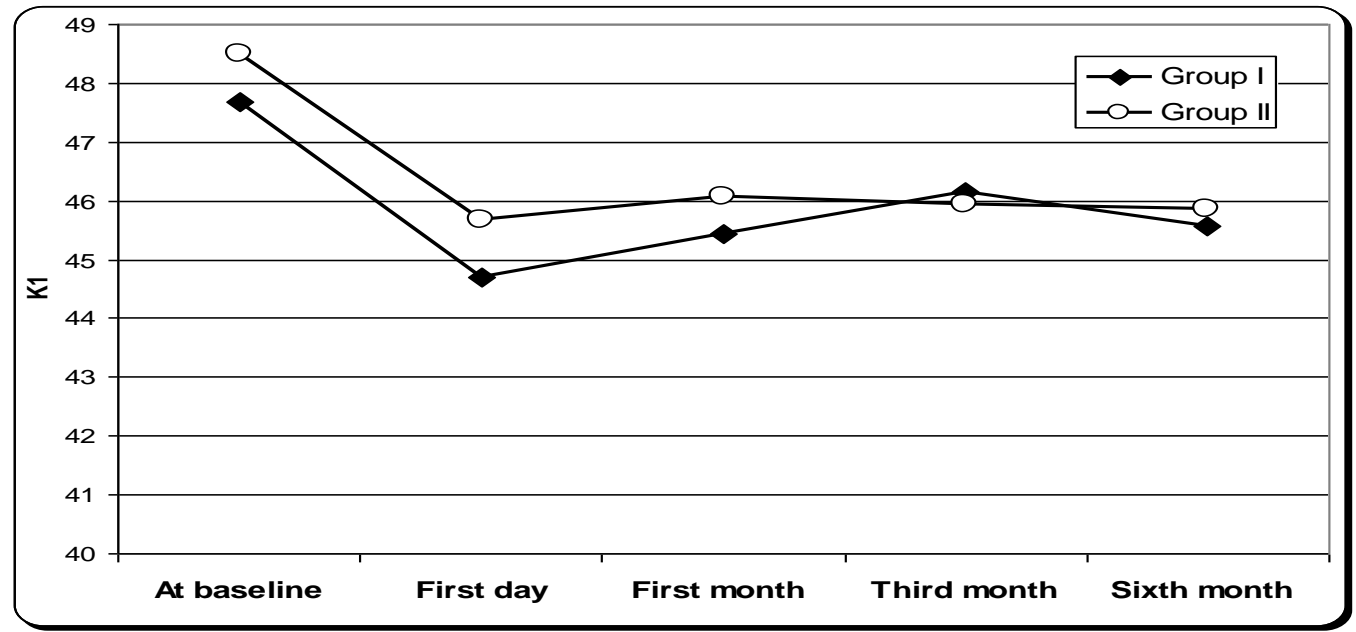

Figure (5). Correlation between K1 after intracorneal ring implantation in both groups.

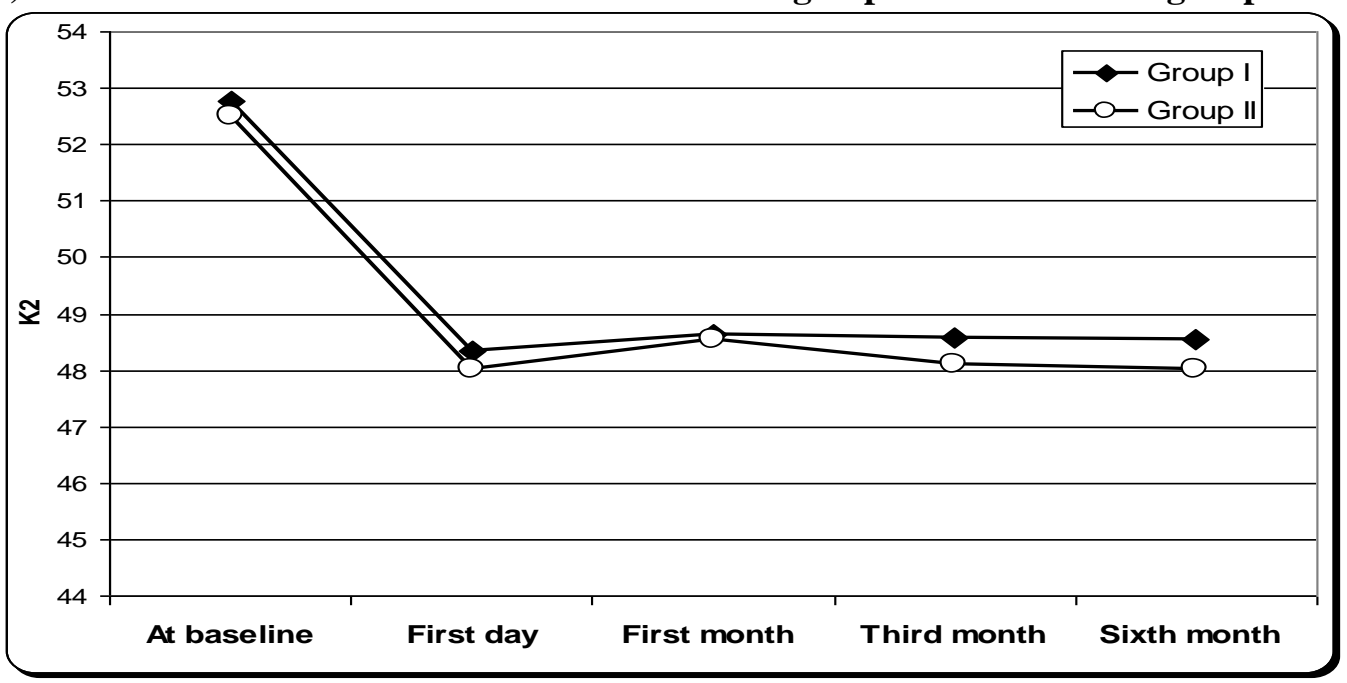

Figure (6). Correlation between K2 after intracorneal ring implantation in both groups. 
Visual Acuity, Rafraction, And K Reading Changes After Five Versus...

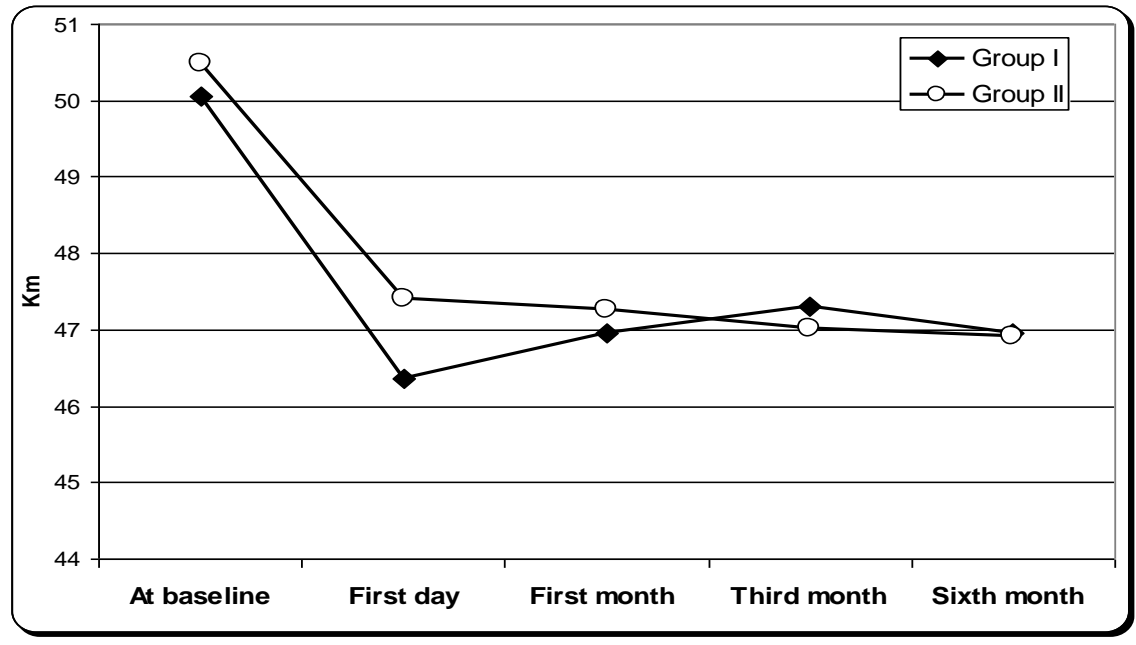

Figure (7). Correlation between UCVA after intracorneal ring implantation in both groups.

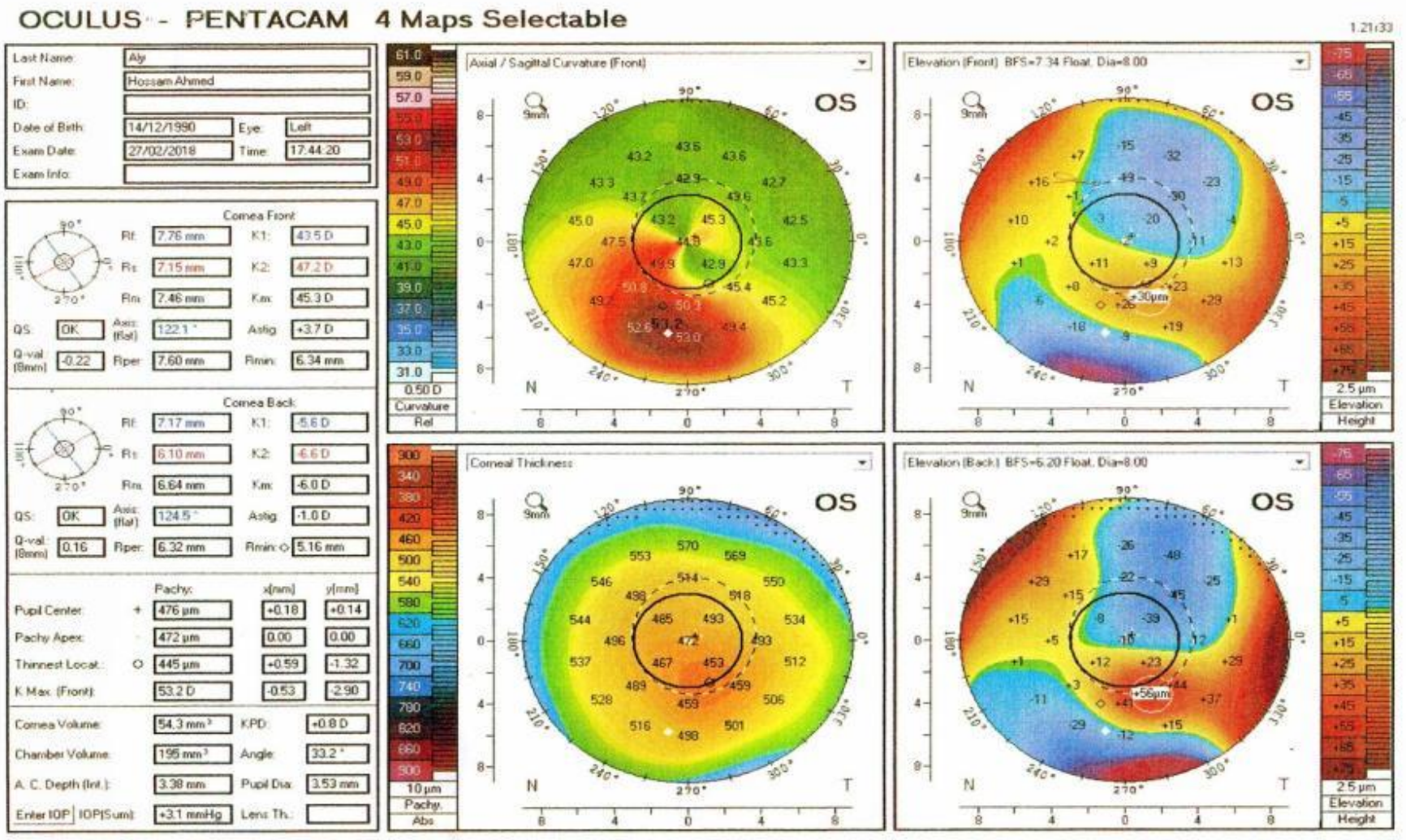


OCULUS - PENTACAM 4 Maps Selectable
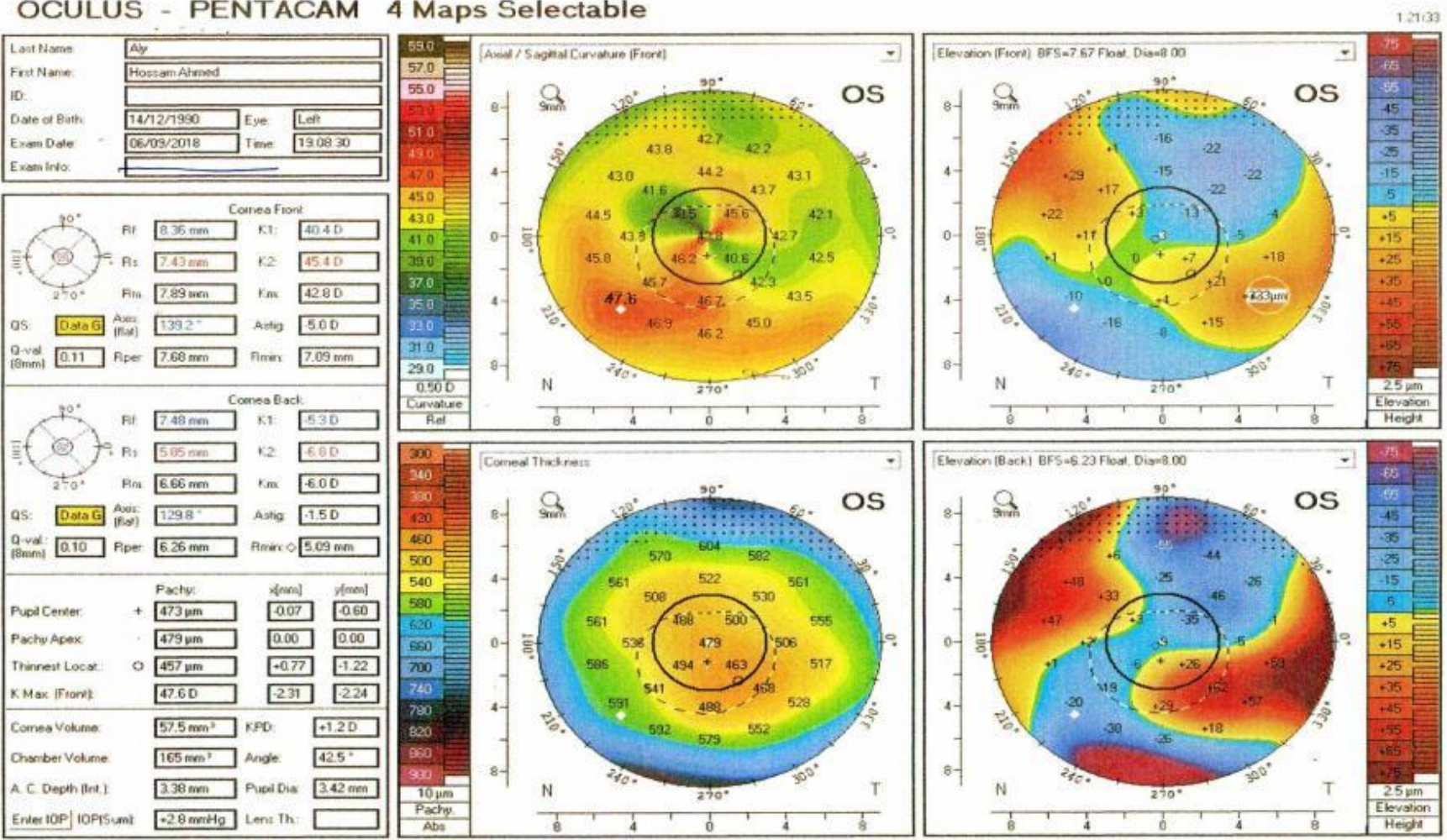

Figure (8). Pre and postoperative pentacam of patient no. 5 in group I.

WAVELIGHT - ALLEGRO OCULYZER
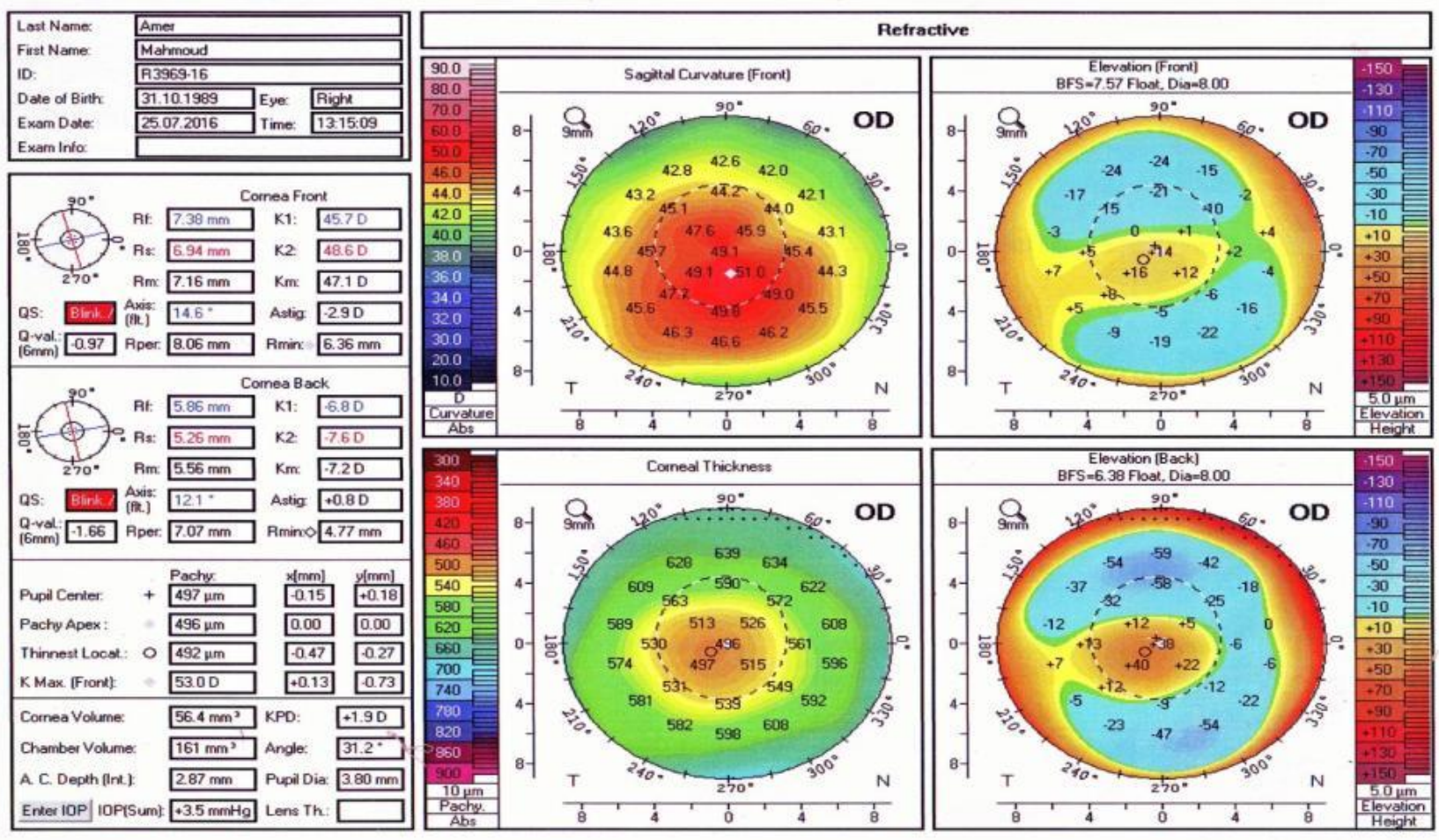

Pre and postoperative pentacam of patient no5 in group (1) 
WAVELIGHT - ALLEGRO OCULYZER
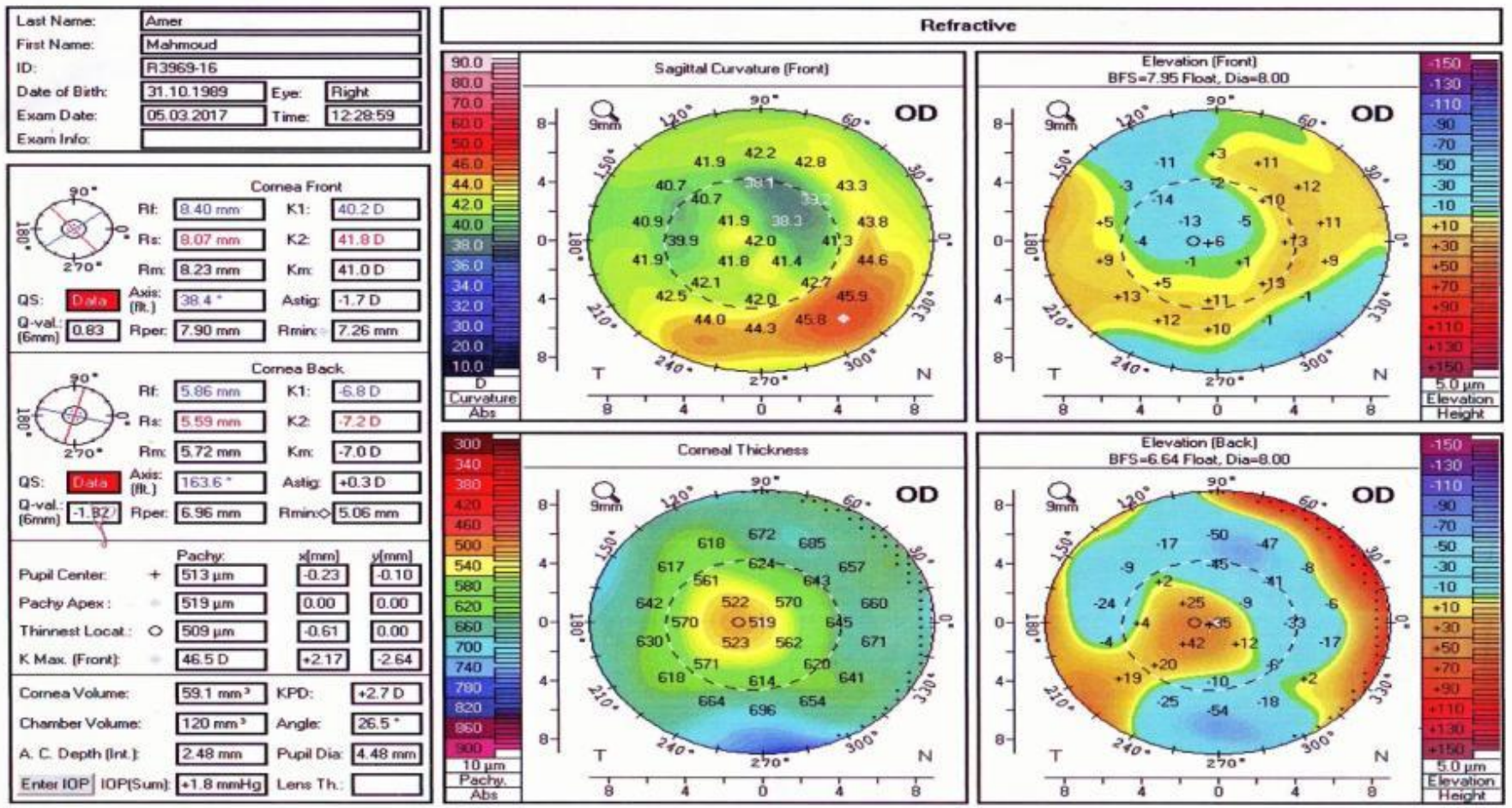

Figure (9). Pre and postoperative pentacam of patient no. 10 in group II

\section{Discussion}

The results of the present study showed a significant improvement in spherical errors, cylindrical errors, UCVA, BCVA as well as K readings after keraring segment implantation in keratoconus during the follow-up visits. The present study showed that femtosecond-assisted intracorneal implantation improved visual acuity and refraction, and decreased $\mathrm{K}$ readings after the procedure in patients with grades (2-4) of keratoconus. The results showed a significant improvement of $\mathrm{K}$ readings, spherical errors, cylindrical errors as well as UCVA and BCVA. The safety, efficacy, and predictability of the procedure were acceptable and in line with other studies. In group I, k1, $\mathrm{k} 2$ and $\mathrm{Km}$ were improved. This correlates with the study done by Mirazaei and colleagues $^{(4)}$ on 30 eyes of 21 keratoconus. They reported preoperative a clinically significant reduction in mean keratometry $(\mathrm{p}=0.000)$. In group II, $\mathrm{k} 1, \mathrm{k} 2, \mathrm{Km}$ were improved. This correlates with the study done by Miraftab et al. . $^{(5)}$.

In the present study, the mean preoperative spherical and cylindrical errors showed highly statistically significant differences at the follow up periods postoperatively. In the present study, the mean preoperative UCVA and BCVA increased postoperatively.

This agrees with a study published by Seleet $\boldsymbol{e t}$ al. ${ }^{(6)}$ implanted ICRS in 10 eyes of seven patients with keratoconus. All cases were followed up every 3 months for 6 months. An improvement was seen in uncorrected visual acuity $(\mathrm{P} \leq 0.05)$, best spectacle corrected visual acuity $(\mathrm{P} \leq 0.001)$. Keraring implantation is highly effective in reducing both myopic and astigmatic errors. A study done by Puell and Alvarez. ${ }^{(7)}$ reported that 27 eyes of 27 subjects with keratoconus were included, follow up after 6 months ICR implanted with optical zone $(5 \mathrm{~mm})$, the mean UCVA was $1.21 \pm 0.46$. It was improved to $0.75 \pm 0.40$ postoperatively. Mean BCVA was $0.42 \pm 0.28$ was improved to $0.24 \pm 0.15 \log$ MAR $(\mathrm{p}<0.01)$. Mean sphere was $-4.35 \pm 5.19 \mathrm{D}$. It was decreased to $-2.54 \pm 4.49 \mathrm{D}$. Mean cylinder was $5.07 \pm 2.77 \mathrm{D}$. It was decreased to $-2.81+2.20 \mathrm{D}$. Mean SE-6.89+5.80D was decreased to $3.74 \pm 4.43 \mathrm{D}$ postoperatively.

In our study, significant differences between both groups were noted when comparing 
preoperative and postoperative cylindrical errors and BCVA. One case with postoperative extruded the ring in patient no. 17 in group I. There were no intraoperative complications.

This study disagrees with the study reported by Kaya et al. ${ }^{(8)}$ who performed a retrospective study, 16 eyes that had been implanted with Intacs ICRS (Intacs group) with internal diameter of $6.77 \mathrm{~mm}$, and 17 eyes were implanted with Ferrara ICRS with an internal diameter of $4.40 \mathrm{~mm}$ (Ferrara group). They reported a significant decrease in spherical equivalent refractive error of $3.76 \pm 0.39$ diopters, and $3.42 \pm 0.88 \mathrm{D}$ and keratometry of $3.43 \pm 0.24$ $\mathrm{D}$ and $3.28 \pm 0.78 \mathrm{D}$ in the Intacs and Ferrara groups, respectively; and increase in mean UDVA and CDVA in Snellen lines of $0.18 \pm 0.04$ and $0.21 \pm 0.05$, respectively, in the Intacs group and $0.21 \pm 0.09$ and $0.26 \pm 0.08$, respectively, in the Ferrara group. The postoperative increase in UDVA and CDVA and decrease in keratometry readings were statistically insignificantly between both groups $(\mathrm{P}>0.05$ for all).

\section{Conclusion}

- Both $5 \mathrm{~mm}$ and $6 \mathrm{~mm}$ ICRS improve significantly UCVA, BCVA, decrease the corneal power and corneal astigmatism, but $6 \mathrm{~mm}$ showed significant improvement in cylindrical errors and BCVA as compared to $5 \mathrm{~mm}$ ICR.

\section{References:}

1- Burdon KP, Vincent AL (2013): Insights into keratoconus from a genetic perspective. Clin Experiment Ophtalmol., 96(2):146-54.
2- Haddad W, Fadlallah A, Dirani A et al. (2012): Comparison of 2 types of intrastromal corneal ring segments for keratoconus. J Cataract Refract Surg., 38:1214-1221.

3- Hosny M, El-Mayah E, Sidky MK et al.(2015): Femtosecond laser-assisted implantation of complete versus incomplete rings for keratoconus treatment. Clin Ophthalmol., 9: 121-127.

4- Mirazaei M, Amin $N$, Kurosh $S$ et al.(2014): Clinical and Topographical Corneal Changes after Keraring Implantation in Keratoconus Patients. Advances in Bioscience \& Clinical Medicine, 3(1):40-45.

5- Miraftab M, Hashemi H, Hafezi F et al. (2016): Mid-Term Results of a Single Intrastromal Corneal Ring Segment for Mild to Moderate Progressive Keratoconus.Cornea,10(5):1-5.

6- Seleet MM, Soliman AH, Alaaeldin OM (2015): Femtosecond laser intracorneal ring segment implantation based on a nomogram modification in type 1 and type 2 ectasia. J Egypt Ophthalmol Soc., 108:1-5.

7- Puell MC and Alvarez CJ (2016): Forward light scatter and visual acuity before and after intrastromal corneal ring segment implantation at different stages of keratoconus Acta Ophthalmol., 94: 738-743.

8- Kaya V, Utine CA, Karakus $S \mathrm{H}$ et al. (2011): Refractive and Visual Outcomes After Intacs vs Ferrara Intrastromal Corneal Ring Segment Implantation for Keratoconus: A Comparative Study. J Refract Surg., 27(12):907-912. 